\title{
Monte Carlo Modeling of YBCO Vapor Deposition
}

\author{
Jing Fan", Iain D. Boyd", and Chris Shelton ${ }^{\dagger}$ \\ Department of Aerospace Engineering, University of Michigan, Ann Arbor, MI 48109* \\ $3 M$ Corporate Process Technology, St. Paul, MN 55144
}

\begin{abstract}
A three dimensional electron beam physical vapor deposition process of super-conducting films of $\mathrm{YBa}_{2} \mathrm{Cu}_{3} \mathrm{O}_{7-\delta}$ in a vacuum chamber is investigated both computationally and experimentally. The numerical analysis employs the direct simulation Monte Carlo (DSMC) method. Some important modeling issues such as atomic collision cross-sections for metal vapors and hyperfine electronic structure of the atomic absorption spectra are addressed. Film deposition thickness profiles and atomic absorption spectra given by the DSMC method and experiment for pure yttrium evaporation are in excellent agreement. Interaction between the vapor jets is found to have a significant effect on the film growth rate and species molar ratio that are key factors of the film technology.
\end{abstract}

\section{INTRODUCTION}

Electron Beam Physical Vapor Deposition (EBPVD) is an important deposition technique for a variety of materials that involve the use of an electron beam to vaporize metallic atoms from a solid ingot. Applications of this technology include coating of high temperature super-conducting films, metallic coating of fibers, and enrichment of uranium for nuclear power generation. A critical component of these processes is the manner in which the atoms are transported from the ingot to the deposition surface. There is a need to understand in detail the gas dynamics of the expansion process particularly in cases where multiple ingots of different species are evaporated.

In this paper, we consider both numerically and experimentally the deposition of super-conducting films of $\mathrm{YBa}_{2} \mathrm{Cu}_{3} \mathrm{O}_{7-\delta}$ that are of great technological interest in numerous applications. ${ }^{1}$ Figure 1 shows the top and side views of the experimental deposition chamber. Using vacuum pumps, the background pressure is maintained at $2 \sim 5 \times 10^{-5} \mathrm{Torr}$. In the EBPVD process, atoms are vaporized from the molten surfaces of yttrium, barium and copper ingots by a high-energy electron beam. The vapor jets expand through the chamber, impinge and deposit on a substrate. The vaporization rates achieved with the electron beam produce flow at relatively high density close to the source surfaces. However, as the jets proceed toward the substrate, rapid expansion brings the flow into the noncontinuum regime. The direct simulation Monte Carlo (DSMC) method $^{2}$ is used to simulate the three-dimensional process. Besides the translational energy mode, the atomic electronic energy is taken into account. ${ }^{3}$ At this stage, no flux of oxygen is included.
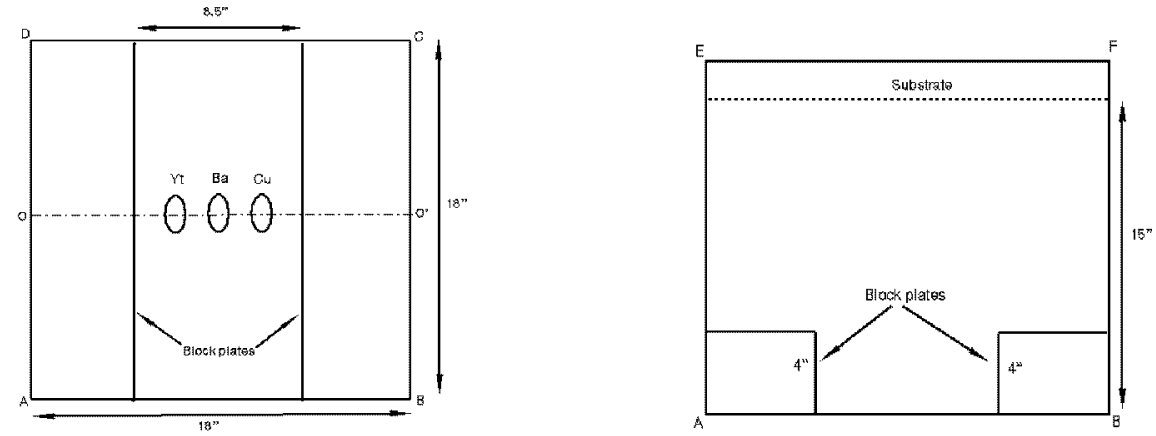

FIGURE 1. Schematic diagrams of the deposition chamber. Left: top view; right: side view.

CP585, Rarefied Gas Dynamics: 22 $2^{\text {nd }}$ International Symposium, edited by T. J. Bartel and M. A. Gallis (C) 2001 American Institute of Physics 0-7354-0025-3/01/\$18.00 


\section{ATOMIC COLLISION CROSS-SECTIONS FOR METAL VAPORS}

The DSMC method is a powerful and general numerical technique for computation of low density, nonequilibrium flows, and has been developed over the past 30 years primarily for aerospace applications. ${ }^{2}$ Application of the DSMC method to metal vapor flows is straightforward in principle and has been demonstrated in relatively simple geometry such as an axisymmetric case. ${ }^{3}$ Some specific complications do naturally arise. One of them is the determination of atomic collision cross-sections for metal vapors. Appropriate collision cross-sections are essential for the DSMC method to simulate particle collisions. They are usually derived from coefficients of viscosity. ${ }^{2,4}$ The measurement of the transport properties for metal vapors at high temperature, however, has proven very difficult because of many unavoidable sources of error. ${ }^{5}$ Actually, except for alkali metal vapors, ${ }^{6-10}$ no viscosity data are available for metal vapors such as yttrium, barium and copper, and few studies theoretically address the collision cross-sections for metal vapors.

The present paper employs the inverse-power model to describe the interaction between atoms of metal vapors

$$
F=\kappa / r^{\eta}
$$

The collision cross-section and coefficient of viscosity for the inverse-power model may be written as ${ }^{4}$

and

$$
\sigma_{T}=\pi W_{0, m}^{2}\left(\frac{\kappa}{m_{r} c_{r}^{2}}\right)^{2 /(\eta-1)}
$$

$$
\mu=\frac{5(k m T / \pi)^{l / 2}(2 k T / \kappa)^{2 /(\eta-l)}}{8 A_{2}(\eta) \Gamma[4-2 /(\eta-1)]},
$$

where $W_{0, m}$ is a constant, $m_{r}$ is the reduced mass, $c_{r}$ is the relative speed, $k$ is the Boltzmann constant, $m$ is the atomic mass, $T$ is the temperature, and $A_{2}(\eta)$ is a numerical factor.

The values of $\eta$ and $\kappa$ for alkali metal vapors have been obtained by equating the right-hand side of Eq. (3) with theoretical data ${ }^{10}$ at temperatures of $700 \mathrm{~K}$ and $2000 \mathrm{~K}$, respectively, and they are given in Table 1 . Figure 2 compares the profiles of Eq. (3) to the theoretical and experimental data for sodium and cesium. The experimental data, ${ }^{3}$ though with scatter, support in trend the theoretical data ${ }^{10}$ and fitted profiles that are in excellent agreement.

TABLE 1. Parameters of the inverse-power model for alkali metal vapors.

\begin{tabular}{lccc}
\hline Element & $\eta$ & $\kappa$ & $\boldsymbol{A}_{2}(\eta)$ \\
\hline Lithium & 6.333 & $7.984 \times 10^{-70}$ & 0.379 \\
Sodium & 7.351 & $4.979 \times 10^{-79}$ & 0.355 \\
Potassium & 6.737 & $4.462 \times 10^{-73}$ & 0.368 \\
Rubidium & 6.669 & $2.521 \times 10^{-72}$ & 0.370 \\
Cesium & 6.531 & $7.081 \times 10^{-71}$ & 0.374 \\
\hline
\end{tabular}
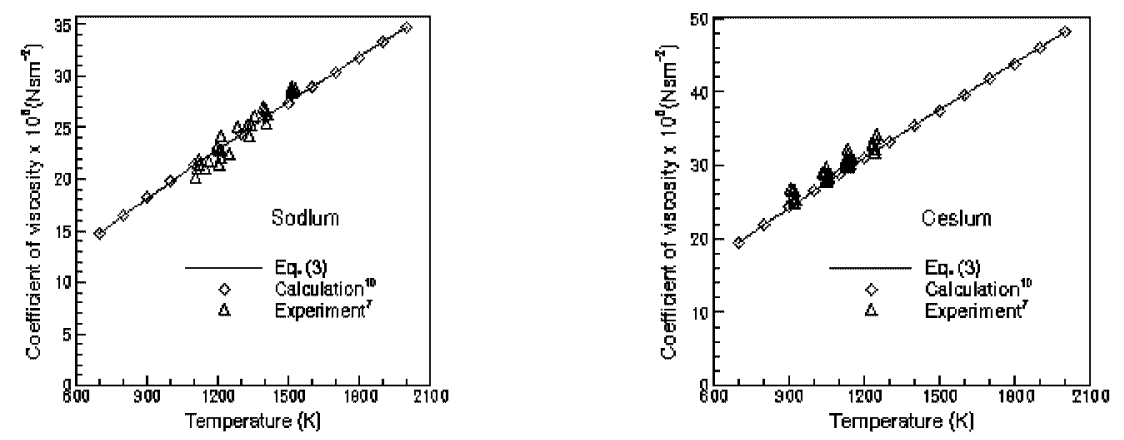

FIGURE 2. Comparison of theoretical, calculated and experimental viscosity for sodium and cesium vapors. 
DSMC calculations often employ the variable hard-sphere (VHS) model ${ }^{2,11}$ to describe the interaction between particles. It assumes that the scattering during particle collisions is isotropic in the center of mass frame of reference, but the collision cross-section has the same relation with the relative speed as the inverse-power model

$$
\frac{\sigma_{T}}{\pi d_{r e f}^{2}}=\frac{\left(2 k T_{r e f} / m_{r} c_{r}^{2}\right)^{\omega-0.5}}{\Gamma(2.5-\omega)} \text { with } \quad d_{r e f}=\left[\frac{15\left(m k T_{r e f} / \pi\right)^{0.5}}{2(5-2 \omega)(7-2 \omega) \mu_{r e f}}\right]^{0.5},
$$

where $\mu_{\text {ref }}$ is the coefficient of viscosity at a reference temperature $T_{\text {ref }}$, and $\omega=0.5+2 /(\eta-1)$.

The reference collision diameters $d_{\text {ref }}$ for alkali metal vapors have been obtained with $\mu_{\text {ref }}$ calculated using Eq. (3), and they are given in Table 2. We assume that the values of $\eta$ and $\kappa$ for the alkali metal vapors apply to other metal vapors occupying the same row in the periodic table of elements. Based on the assumption, the reference collision diameters for several metal vapors of interest are calculated and given in Table 2 . The selection of $T_{\text {ref }}$ is arbitrary and does not affect collision cross-sections. To be reasonable physically, it is taken to be $2,000 \mathrm{~K}$ that is above the melting point of all elements considered in Table 2.

An outstanding feature of the reference collision diameters for the metal vapors is that they are quite large in comparison with common atomic gases. This is mainly due to the larger viscosity of the gases. For example, the coefficient of viscosity for argon gas is $5.452 \times 10^{-5} \mathrm{Nms}^{-2}$ at $700 \mathrm{~K}$ and $0.1 \mathrm{MPa},{ }^{12}$ while the viscosity of sodium vapor at the same conditions is $1.475 \times 10^{-5} \mathrm{Nms}^{-2} .10$

TABLE 2. Parameters of the VHS model for metal vapors at $T_{r e f}=2000 \mathrm{~K}$.

\begin{tabular}{lcc} 
Element & $\boldsymbol{d}_{\boldsymbol{r e f}} \times \mathbf{1 0 ^ { - 1 0 }} \boldsymbol{m}$ & $\omega$ \\
\hline Lithium & 4.562 & 0.875 \\
Sodium & 4.961 & 0.815 \\
Potassium & 5.554 & 0.849 \\
Rubidium & 5.846 & 0.853 \\
Cesium & 6.306 & 0.862 \\
Copper & 6.272 & 0.849 \\
Titanium & 5.844 & 0.849 \\
Yttrium & 5.903 & 0.853 \\
Zirconium & 5.941 & 0.853 \\
Barium & 6.358 & 0.862 \\
\hline
\end{tabular}

\section{ATOMIC ABSORPTION SPECTRA}

In situ monitoring and control is one of the primary issues involved in EBPVD processing of super-conducting films of $\mathrm{YBa}_{2} \mathrm{Cu}_{3} \mathrm{O}_{7-\delta}$. Diode-laser-based absorption monitors for yttrium and barium have been developed to meet this need.13 They are simultaneously both element-specific and non-invasive, and can provide the atomic absorption spectra from which the atomic flux across the laser path may be derived.

Using the monitors, an atomic absorption spectral distribution around a central frequency $v_{0}$ along a laser path is observed. The spectrum $I(v)$ in the vicinity of $v_{0}$ is called the line profile. ${ }^{14}$ The profile may have many peaks referred to as hyperfine electronic structure. They correspond to the frequencies absorbed by particles when the light beam traverses the subject material, and are centered at $v_{i}=v_{0}+\Delta v_{i}\left(\Delta v_{i} \ll<v_{0}\right)$. These peaks have a finite width due to various effects of broadening. In low pressure $\left(10^{-2}\right.$ Torr $)$ situations, the width is mainly determined by the Doppler effect. ${ }^{14}$ This takes place because frequencies absorbed by particles with different velocities in the light beam direction are slightly different.

At thermal equilibrium, a Doppler-broadening peak shape centered at a frequency $v_{i}$ is described by ${ }^{14}$ 


$$
I_{i}(v)=\frac{\beta \lambda_{i}}{\sqrt{\pi}} \exp \left[-\beta^{2} \lambda_{i}^{2}\left(v-v_{i}\right)^{2}\right],
$$

where the wavelength $\lambda_{i}=c / v_{i}, c$ is the light speed, and $\beta=\sqrt{m / 2 k T}$. The spectral distribution (5) corresponds to a Maxwellian velocity distribution

$$
f\left(v_{\ell}\right)=\frac{\beta}{\sqrt{\pi}} \exp \left(\beta^{2} v_{\ell}^{2}\right)
$$

where $v_{\ell}$ is the thermal velocity component in the light-beam direction. Comparing the distributions (5) and (6) leads to the relations

$$
v-v_{i}=v_{\ell} / \lambda_{i}
$$

and

$$
I_{i}(v)=\lambda_{i} f\left(v_{\ell}\right) \text {. }
$$

A velocity distribution of particles may be obtained from DSMC calculations. To compare with an experimental absorption spectrum, it needs to be converted into an absorption scan using equations (7) and (8). Before the conversion, we must normalize the calculated velocity distribution $f\left(v_{\ell}\right)$ in accordance with the measured absorption spectrum $I_{i}(v)$. The normalization condition requires

$$
A_{v_{\ell}} \int_{\Omega_{v_{\ell}}} f\left(v_{\ell}\right) v_{\ell}^{*-1} d v_{\ell}=1
$$

and

$$
B_{v} \int_{\Omega_{v}} I(v) v^{*-1} d v=1
$$

where $v_{\ell}^{*}$ and $v^{*}$ are velocity and frequency normalization factors, respectively, and $A_{v_{\ell}}$ and $B_{v}$ are overall normalization factors. The relation (7) requires

$$
v^{*}=v_{\ell}^{*} / \lambda_{i}
$$

To compare calculated and experimental atomic absorption spectra properly, hyperfine electronic structure must be included. ${ }^{14,15}$ For example, an experimental profile line for the ground state transition of yttrium at $v_{0}=4.486 \times 10^{14} \mathrm{~Hz}$ consists of four peak components with $\Delta v_{i}$ and relative intensity factors $\gamma_{i}$ given in the second column of Table 3. Accordingly, the calculated line profile must also be the weighted sum of the four peak shapes centered at slightly different frequencies $v_{i}=v_{0}+\Delta v_{i}$

$$
I^{c a l}(v)=\sum_{i=l}^{4} \gamma_{i} I_{i}^{c a l}(v) / \sum_{i=l}^{4} \gamma_{i}
$$

TABLE 3. Hyperfine structure parameters of atomic absorption spectra for yttrium.

$\begin{array}{ccc}v_{0} \times 10^{14}(\mathrm{~Hz}) & 4.486 & 4.416 \\ \Delta v_{1} \times 10^{8}(\mathrm{~Hz}) & 0.00 & 0.00 \\ \Delta v_{2} \times 10^{8}(\mathrm{~Hz}) & 1.14 & 0.86 \\ \Delta v_{3} \times 10^{8}(\mathrm{~Hz}) & 1.76 & 1.99 \\ \Delta v_{4} \times 10^{8}(\mathrm{~Hz}) & 2.90 & 1.99 \\ \gamma_{1} & 5 & 20 \\ \gamma_{1} & 1 & 1 \\ \gamma_{1} & 1 & 1 \\ \gamma_{4} & 9 & 4\end{array}$




\section{RESULTS AND DISCUSSION}

Two cases are considered here. For the first case where only the yttrium source is evaporating, the evaporation rates are measured by the feed rate of the ingot into the chamber (Table 4). The source surface temperatures $T_{s}$ are calculated from ${ }^{16}$

$$
\log _{10} \Gamma_{g}=C-0.5 \log _{10} T_{s}-B / T_{s},
$$

where $\Gamma_{g}$ denotes the evaporation rate in unit of $\mathrm{g} / \mathrm{cm}^{2} \mathrm{sec}$. The values of $B$ and $C$ for many metal vapors including yttrium, barium and copper are given in Table 10.2 in Ref. 16. The number densities at the source surfaces are calculated from $n=2 \sqrt{\pi} \beta \Gamma_{e}$, where $\Gamma_{e}=\Gamma_{g} / m$, and $\beta=\sqrt{m / 2 k T_{s}}$.

TABLE 4. Source evaporation rates $\Gamma_{e}\left(\right.$ moles $\left./ \mathrm{cm}^{2} \mathrm{sec}\right)$ and surface temperature $\boldsymbol{T}_{s}(\boldsymbol{K})$.

\begin{tabular}{|c|c|c|c|c|c|c|}
\hline \multirow{2}{*}{ Case } & \multicolumn{2}{|c|}{ Yttrium } & \multicolumn{2}{|c|}{ Barium } & \multicolumn{2}{|c|}{ Copper } \\
\hline & $\Gamma_{e}$ & $T_{s}$ & $\Gamma_{e}$ & $T_{s}$ & $\Gamma_{e}$ & $T_{s}$ \\
\hline I & $1.129 \times 10^{-5}$ & 2091 & 0 & - & 0 & - \\
\hline II & $8.542 \times 10^{-6}$ & 2065 & $1.708 \times 10^{-5}$ & 1010 & $2.562 \times 10^{-5}$ & 1732 \\
\hline
\end{tabular}

DSMC calculations are carried out using a software system called MONACO-YBC. ${ }^{17}$ In an orthogonal coordinate system with the origin at $\mathrm{O}$, the $\mathrm{x}$ - and $\mathrm{y}$ - axes are along $\mathrm{OO}^{\prime}$ and $\mathrm{OA}$ respectively, and the $\mathrm{z}$ axis points to the substrate (cf. Fig. 1). The computational grid employs $20 \times 60 \times 50$ cuboid cells. The cell sizes are nonuniform: cell size in the $\mathrm{z}$ direction near the source surfaces are very small to capture the rapid jet expansion, and cell sizes in the $\mathrm{x}$ and $\mathrm{y}$ directions in the region covering the sources are small to resolve the region of flow that is most important for accurate simulation of the deposition process. About two million simulated particles are used in the calculations. The results may be obtained overnight on a SGI OCTANE workstation or a DELL/Pentium III PC.

The chamber walls and block plate surfaces are assumed to be perfectly sticking. This assumption is well satisfied in this study because the wall and plate surface temperatures are low. New particles are vaporized into the chamber from the surfaces of the sources. They may collide with one another above the pool, particularly for cases with high evaporation rates. This causes a fraction of the atoms to develop post-collision velocities that move them back to the molten pools. In Ref. 3, a total back scatter flux was found to be as high as $19 \%$ of the evaporation rate. To maintain a net inflow rate measured by the feed rate of an ingot into the chamber, the back-scattered particles are made to diffusely reflect from the source surface.

\section{Case I: Evaporation of Pure Yttrium}

An experiment of pure yttrium with evaporation rate given in Table 4 is carried out that lasts 12 minutes. Figure 3 compares measured deposition thickness data with DSMC and collisionless profiles. The DSMC and collisionless calculations are carried out using the same code, but the latter turns off atomic collisions. The deposition thickness is measured with a profilometer along a symmetrical line of the substrate. The substrate used is a polished silicon wafer. Since the coating is rather thick and blistering of the film does not allow accurate sampling points, scatter in the measured data is clearly seen. Deducting the measured data scatter, the DSMC profile compares well with the experiment. The collisionless profile is significantly lower than the DSMC and measured results. The atomic collisions impede to some extent the atomic diffusion to the block plates and chamber walls that are assumed to be perfectly sticking. Neglecting these collisions results in the atoms having more chances to stick on the block plate surfaces and chamber walls than to be deposited on the substrate.

Figure 4 shows DSMC and measured atomic absorption (AA) spectra across an aperture close to the symmetric line of the substrate. Because the hyperfine electronic structures of yttrium are different at the frequencies of $668 \mathrm{~nm}$ and $679 \mathrm{~nm}$ (Table 3), the corresponding AA peak structures are inclined to the right and left, respectively. The DSMC and measured Doppler widths and peak-structure details are all in excellent agreement. 
Figure 5 shows the number density given by the DSMC calculation. It shows that there is a rapid expansion around the source that leads to a significant decrease of the number density. Two continuum breakdown criteria, proposed by Bird ${ }^{18}$ and Boyd, et al. ${ }^{19}$, respectively, are computed based on the DSMC number density field. For steady flows Bird's criterion may be written as

$$
P=M a \sqrt{\frac{\pi \gamma}{8}} \frac{\lambda}{\rho}\left|\frac{d \rho}{d s}\right|,
$$

where $M a, \lambda, \rho$ are the local Mach number, mean free path, and density, respectively, $\gamma$ is the specific heat ratio, and $s$ is the distance along a streamline. Studies in Ref. 18 indicated that the continuum approach in expanding flows failed at a value for $P$ of about 0.05 . Comparing Navier-Stokes and DSMC results for shock waves and hypersonic flows around a sphere, Boyd, Chen and Candler ${ }^{19}$ found that the gradient-length local Knudsen number based on density

$$
K n_{G L L-D}=\frac{\lambda|\nabla \rho|}{\rho},
$$

can more precisely specify the limit at which the Navier-Stokes equations were invalid. The continuum approach broke down wherever the value of $K n_{G L L-D}$ exceeded $0.05 .{ }^{19}$ Variation of the two criteria along a path from the yttrium source center perpendicularly to the substrate is showed in Fig. 5. Both of them indicate that the flow is completely in the non-continuum regime that needs to be studied kinetically.
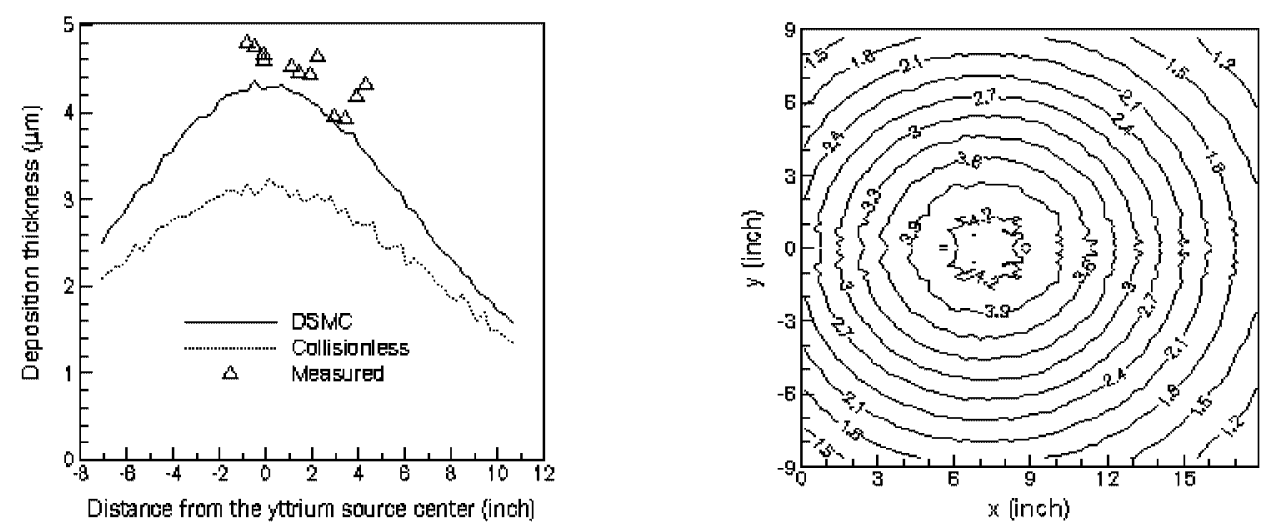

FIGURE 3. Film deposition thickness distributions for Case I. Left: comparison of DSMC, collisionless and measured results along a symmetrical line of the substrate; right: distribution ( $\mu \mathrm{m}$ ) over the whole substrate given by the DSMC calculation.
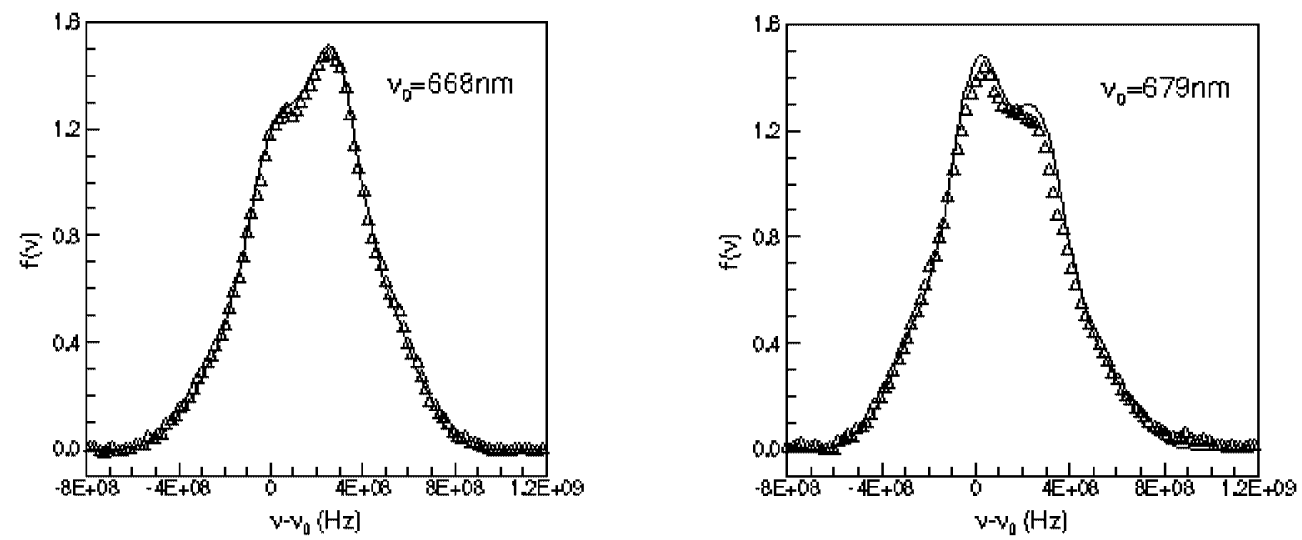

FIGURE 4. Comparison of DSMC (solid line) and measured (triangle) atomic absorption spectra for Case I. Left: at frequency of $4.486 \times 10^{14} \mathrm{~Hz}(668 \mathrm{~nm})$; right: at frequency of $4.416 \times 10^{14} \mathrm{~Hz}(679 \mathrm{~nm})$. 

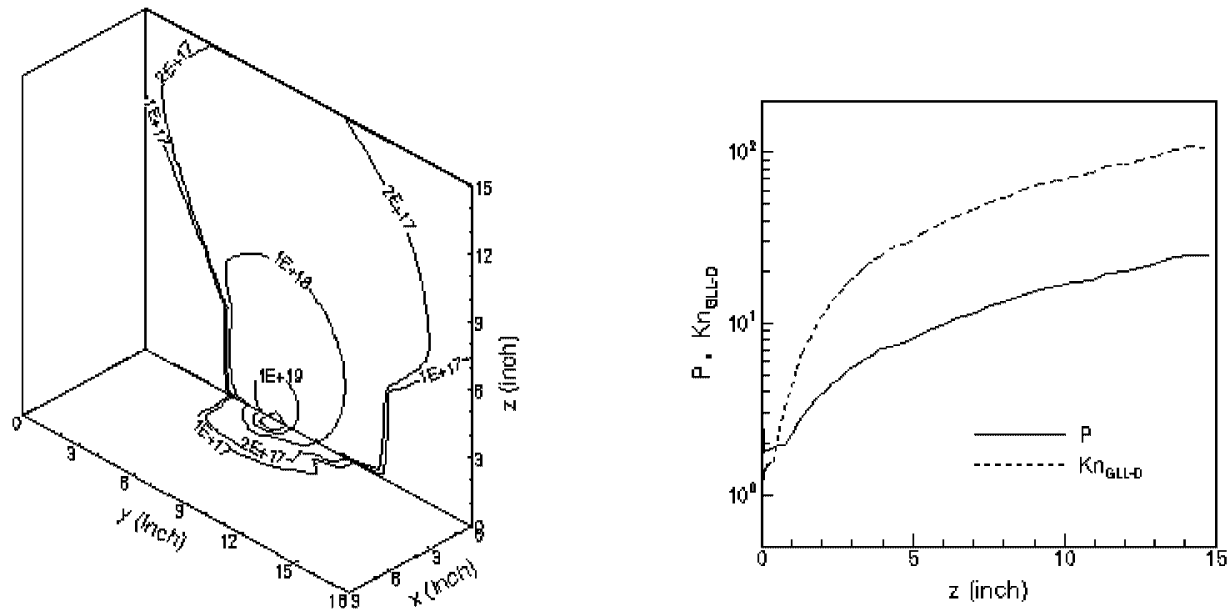

FIGURE 5. Left: DSMC number density field $\left(\mathrm{m}^{-3}\right)$ for Case I; right: variation of two continuum breakdown criteria, derived from the left number density field, along a path from the yttrium source center perpendicularly to the substrate.

\section{Case II: Co-evaporation of Yttrium, Barium and Copper}

The number densities of the yttrium, barium and cooper sources computed from the evaporation rates given in Table 4 are $2.903 \times 10^{20}, 1.032 \times 10^{21}$ and $8.401 \times 10^{20} \mathrm{~m}^{-3}$, respectively. Figure 6 (left) shows the total number density field contours. Similar to Case $I$, there is a significant decrease of the number density around the sources that corresponds to a rapid expansion.
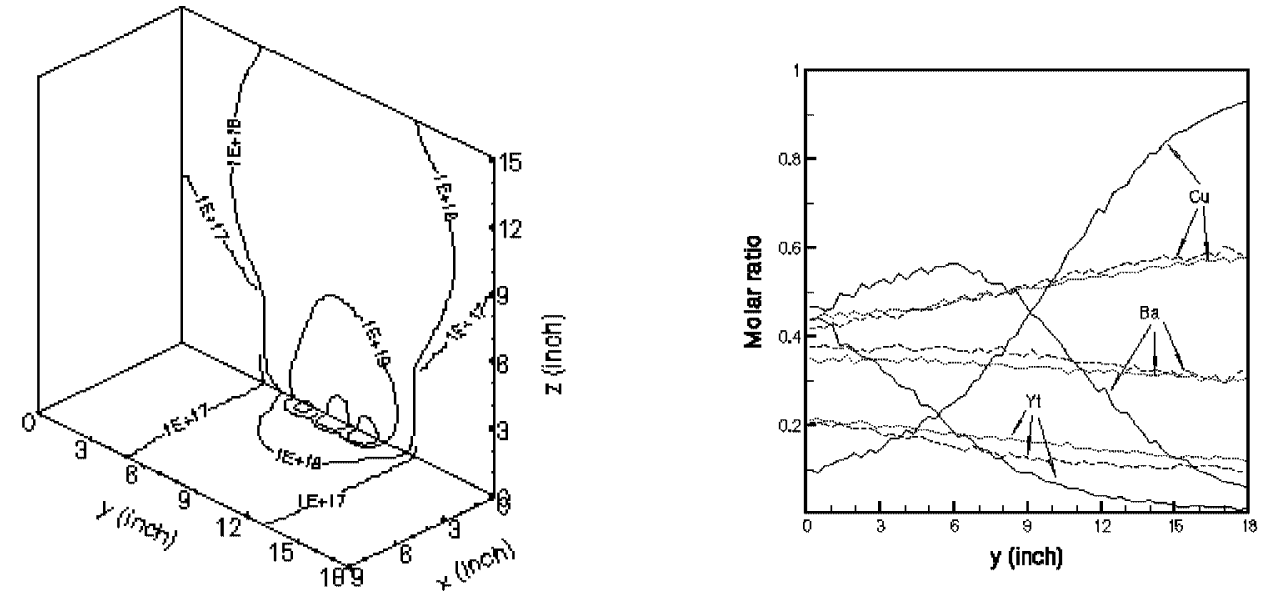

FIGURE 6. DSMC results for Case II. Left: total number density field $\left(\mathrm{m}^{-3}\right)$; right: comparison of species molar ratio along a symmetrical line of the substrate. Collision -, part-collision ---, and collisionless -.-.- .

To understand the effect of collisions between different species, molar species ratios of yttrium, barium, and copper across a symmetrical line of the substrate are compared at collision, part-collision (turning off collisions between the different species), and collisionless situations (Fig. 6, right). The part-collision profiles are close to the collisionless profiles that are relatively uniform and keep a ratio near to the original one between the yttrium barium, and copper sources, i.e., 1: 2: 3. Because the collisions between yttrium and barium or copper atoms generally impede a diffusion of the yttrium atoms in the positive y-direction, the collision profile of yttrium is significantly non-uniform and peaks at $y=0$. A similar mechanism results in a peak at $y=18^{\prime \prime}$ in the collision profile of copper. 
Since the number density of the copper source is about three times that of the yttrium, the collisions between barium and copper atoms are more frequent than those between barium and yttrium atoms. This causes the barium peak to be inclined to the left. The significantly non-uniform distributions of the molar ratio are actually deleterious to the quality of super-conducting films of $\mathrm{YBa}_{2} \mathrm{Cu}_{3} \mathrm{O}_{7-\delta}$ that requires a ratio of 1:2:3 between yttrium, barium and copper. The interaction between the vapor jets revealed by the calculations is helpful to improve the film technology.

\section{CONCLUSIONS}

A three dimensional electron beam physical deposition of super-conducting films of $\mathrm{YBa}_{2} \mathrm{Cu}_{3} \mathrm{O}_{7-\delta}$ was studied using the DSMC method and experiment. Atomic collision cross-sections for metal vapors such as yttrium, barium and copper were given. They were quite large in comparison with those for common atomic gases. A means to convert a velocity distribution of atoms into an absorption spectrum was established. Film deposition thickness profiles along a symmetrical line of the substrate and atomic absorption spectra across an aperture close to the symmetrical line given by the DSMC method and experiment for pure yttrium evaporation were in excellent agreement. Interaction between the vapor jets was found to have a significant effect on the film growth rate and species molar ratio that are key factors of the film technology.

\section{ACKNOWLEDGMENTS}

This work was developed as part of the office of Naval Research/3M "Models, Sensors, and Controls for E-Beam Deposition" program, Agreement No. N00014-98-3-0015. The content does not necessarily reflect the position or policy of the Government and no official endorsement should be inferred.

\section{REFERENCES}

1. Chi, C.C. and Dover, R.B., High Tc Supercoductivity Thin Films and Applications, SPIE, Washington, 1990.

2. Bird, G.A., Molecular Gas Dynamics and the Direct Simulation of Gas Flows, Clarendon Press, Oxford, 1994.

3. Balakrishnan, J., Boyd, I.D., and Braun, D.G., Journal of Vacuum Science and Technology A 18, 907-916 (2000).

4. Chapman, S., and Cowling, T.G., The Mathematical Theory of Non-Uniform Gases, Cambridge Univ. Press, London, 1952.

5. Wakeham, W.A., Nagashima, A., and Sengers, J.V., Measurement of the Transport Properties of Fluids, Vol. III, IUPAC/Blackwell Scientific Press, London, 1991.

6. Vargaftik, N.B., and Yargin, V.S., "Thermal Conductivity and Viscosity of the Gaseous Phase", in Handbook of Thermodynamic and Transport Properties of Alkali Metals, edited by R.W.Ohse, Blackwell, London, 1985.

7. Vargaftik, N.B., Vinogradov, V.S., Dolgov, V.I., Dzis, V.G., Stepanenko, I.F., Yakimovich, Y.K., Yargin, V.S., International Journal of Thermodynamics 12, 85-103 (1991).

8. Neoto de Cartro, C.A., Fareleira, J.M.N.A., Matias, P.M., Ramires, M.L.V., Canelas, A.A.C., Varandas, A.J.C., Ber. Bunsenges. Phys. Chem. 94, 53-59 (1990).

9. Fialho, P.S., Ramires, M.L.V., Fareleira, J.M.N.A., Neoto de Cartro, C.A., Ber. Bunsenges. Phys. Chem. 97, 1487-92 (1994).

10. Fialho, P.S., Ramires, M.L.V., Neoto de Cartro, C.A., Fareleira, J.M.N.A., Mardolcar, U.V., Ber. Bunsenges. Phys. Chem. 98 , 92-102 (1994).

11. Bird, G.A., "Monte Carlo Simulation in an Engineering Context", in Progress in Astronautics and Aeronautics, Vol. 74, 239-255 (1981).

12. Kestin, J., and Wakeham, W.A., Transport Properties of Fluids: Thermal Conductivity, Viscosity, and Diffusion Coefficient, Hemisphere, New York, 1988.

13. Wang, W., Hammond, R.H., Fejer, M.M., Beasley, M.R., Journal of Vacuum Science and Technology 17, $2676-2684,1999$.

14. Demtroder, W. Laser Spectroscopy: Basic Concepts and Instrumentation, $2^{\text {nd }}$ enlarged edition, Springer, 1996.

15. Wang, W., Hammond, R.H., Fejer, M.M., Arnason, S., Beasley, M.R., Applied Physics Letters 71, 31 -33 (1997).

16. Dushman, S., Scientific Foundation of Vacuum Technology, John Wiley \& Sons, New York, 1962.

17. Fan, J., and Boyd, I.D., The MONACO-YBC System for Simulating E-Beam Deposition, Release Version 1.0, The University of Michigan, Ann Arbor, January, 2000.

18. Bird, G.A., AIAA Journal 8, 1998-2003 (1970).

19. Boyd, I.D., Chen, G., and Candler, G.V., Physics of Fluids 7, 210-219 (1995). 